\title{
Praxis de la innovación educativa a través de YouTube en tiempos de pandemia \\ Praxis of educational innovation through YouTube in times of pandemic
}

\author{
Carlos Oliva Marañón ${ }^{1}$, Sara Gallego Trijueque ${ }^{2}$ \\ carlos.oliva.maranon@urjc.es, sara.gallego@urjc.es \\ ${ }^{1}$ Comunicación Audiovisual y Publicidad \\ Universidad Rey Juan Carlos \\ Madrid, España \\ ${ }^{2}$ Ciencias de la Comunicación y Sociología \\ Universidad Rey Juan Carlos \\ Madrid, España
}

\begin{abstract}
Resumen- YouTube se ha convertido en una plataforma audiovisual vinculada a los procesos de enseñanza-aprendizaje. Los objetivos de esta práctica de innovación educativa son mejorar el aprendizaje del alumnado, fomentar un acercamiento al uso de herramientas digitales dentro del aula entre los alumnos y estimular su motivación, generando interacción y colaboración de forma grupal. A través de YouTube, los docentes seleccionan un conjunto de materiales audiovisuales relativos a campañas publicitarias y películas para ser analizados por el alumno, mediante una praxis metodológica dividida en fases para fomentar el aprendizaje de forma progresiva. El universo muestral está compuesto por 158 alumnos de la Universidad Rey Juan Carlos y la metodología se ha aplicado a las asignaturas "Sociología de la Desviación" y "Procesos de la Comunicación en Publicidad, Relaciones Públicas y Audiovisual". A tenor de los resultados obtenidos, se constata que la experiencia de innovación docente realizada por los discentes fue útil y beneficiosa, generando motivación y cooperación entre ellos, además de fomentar su capacidad crítica y de abstracción. Se propone, a raíz de los excelentes resultados académicos obtenidos, la continuidad de la aplicación de esta metodología para el desarrollo de las materias mencionadas en cursos venideros.
\end{abstract}

Palabras clave: Youtube, Innovación Docente, Sociología de la Desviación, Procesos de la Comunicación en Publicidad, Relaciones Públicas y Audiovisual, Espacio Europeo de Educación Superior (EEES).

Abstract- YouTube has become an audiovisual platform linked to teaching-learning processes. The objectives of this practice of educational innovation are to improve student learning, encourage an approach to the use of digital tools within the classroom among students and stimulate their motivation, generating group interaction and collaboration. Through YouTube, teachers select a set of audiovisual materials related to advertising campaigns and films to be analyzed by the student through a methodological practiced divided into phases to promote learning progressively. The sample universe is made up of 158 students from the Rey Juan Carlos University and the methodology has been applied to the subjects "Sociology of Deviation" and "Communication Processes in Advertising, Public Relations and Audiovisual". Based on the results obtained, it is found that the teaching innovation experience carried out by the students was useful and beneficial, generating motivation and cooperation among them, in addition to fostering their critical and abstract capacity. It is proposed, as a result of the excellent academic results obtained, the continuity of the application of this methodology for the development of the subjects mentioned in future courses.
Keywords: YouTube, Teaching Innovation, Sociology of Deviance, Communication Processes in Advertising, Public Relations and Audiovisual, European Higher Education Area (EHEA).

\section{INTRODUCCIÓN}

En tiempos de pandemia, es un hecho que en la educación superior se utilicen diferentes herramientas tecnológicas, con el fin de mejorar el aprendizaje del alumnado, ya que es aplicado para ofrecer una enseñanza online más motivadora y pedagógica. Una de las herramientas que ya es habitual utilizar en la educación superior es YouTube. En un estudio realizado por Gallego y Murillo en 2018, se realizaron 124 cuestionarios a diferentes alumnos de los diversos grados impartidos en la Facultad de Educación de la Universidad de Sevilla, concluyendo que YouTube se considera "como una herramienta educativa con un alto potencial de uso en la práctica diaria de clase, por sus múltiples ventajas metodológicas relacionadas con la innovación, aumento de la motivación, potenciación de ampliar y afianzar conocimientos e información, y la integración de las TIC en los procesos educativos".

En la misma línea, Mazara entiende que "los videos son prácticos para la información, ya que no solo poseen funciones informativas, sino que también poseen funciones motivadoras, introductorias, instructivas y de recapitulación" (MarazaQuispe, et al., 2020).

En esta investigación se realizará un estudio de YouTube como plataforma de innovación educativa en las asignaturas de "Sociología de la Desviación" y "Procesos de la Comunicación en Publicidad, Relaciones Públicas y Audiovisual", a través de diferentes prácticas realizadas por los alumnos.

La sociología de la desviación y la criminología son disciplinas afines. La sociología contribuye a que el alumno del Grado en Criminología analice el comportamiento delictivo como un hecho social, sin centrarse únicamente en las particularidades psicológicas o en la parte más criminalística del hecho. De esta forma, la Sociología de la Desviación facilita los discernimientos precisos para poder entender y vislumbrar los factores y causas sociales directamente relacionados con la conducta desviada, contextualizando estos comportamientos desviados dentro de una sociedad y cultura determinada. 
En la misma línea epistemológica, (Fanjul y Morales, 2017, p.19) entienden que la Sociología de la Desviación "se encarga del estudio del comportamiento humano que se aleja de lo socialmente esperado y aceptado y que conforma el conjunto de conductas que son reconocidas como desviadas".

El objetivo de la Sociología de la Desviación es el análisis del sujeto, colectivo social y organización normativa, en base a estos tres elementos se aspira a entender "la génesis delictiva, las consecuencias que tiene el delito en el autor, en las víctimas y en el entorno y cómo este, en el sentido más amplio, puede facilitar su aparición" (Vicente, 2011, p.31). Según Becker (2010, p.21), otro de los objetivos más importantes de la Sociología de la Desviación es el estudio de "las situaciones de infracción y aplicación de la regla, y los mecanismos que hacen que algunas personas rompan las reglas y otros las impongan”.

En resumen, la Sociología de la Desviación “es un campo amplio en el que caben toda clase de fenómenos sociales que impliquen algún tipo de ruptura de la convención, conformidad o de la norma" (Riviére, 2013, p.39).

En relación con la sociología, desde la génesis de la humanidad, el hombre, atendiendo a su caracterización como un ser social, siempre ha sentido la necesidad de comunicarse y relacionarse con otros individuos. En estos procesos comunicativos que, en multitud de ocasiones, se realizan a través de los Medios de Comunicación Social (MCS), intervienen diferentes elementos en el proceso de la comunicación: emisor, mensaje, canal y receptor. Además, hay que tener en cuenta que esos mensajes llegan a una audiencia heterogénea, por lo que el profesional de la información tiene que expresarse con un lenguaje claro para que la información la comprendan todos los ciudadanos, independientemente de su nivel cultural.

Sirva como paradigma de modelo de comunicación, la propuesta realizada por Lasswell en 1948, quien describe un acto de comunicación que responde a las preguntas: quién (comunicador), dice qué (mensaje), en qué canal (medio), a quién (receptor) y con qué efecto (efectos) (McQuail y Windahl, 1997). (Citado en Muela, 2019, p. 13).

En lo concerniente a la publicidad, hay que reseñar que es casi tan antigua como la humanidad, ya que la teoría más aceptada acerca del primer anuncio publicitario es el Papiro de Tebas, encontrado hace tres mil años en Egipto. "Aquí se encuentra el que se considera el primer anuncio de la historia que se conserva. Se trata de un texto sobre Shem, un esclavo desaparecido, por el que Hapú, el tejedor, ofrece a quien lo encuentre una recompensa. El aviso termina así: La Casa de Hapú ofrece las mejores telas de Tebas". (CNICE).

Por lo que respecta al concepto de publicidad, Muela asevera: "La publicidad es una comunicación persuasiva que financia los medios en los que trabajan los periodistas, muchos de los cuales incluso colaboran en la realización de campañas" (Muela, 2019, p. 9). Igualmente, Muela afirma que "muchos periodistas desarrollan su actividad profesional en el medio audiovisual, por lo que un acercamiento al proceso de comunicación radiofónico, televisivo o cinematográfico les permitirá descubrir esos puntos de conexión para valorar una opción laboral en ese ámbito” (Muela, 2019, p. 10).

La asignatura "Procesos de Comunicación en Publicidad, Relaciones Públicas y Audiovisual" "responde al necesario conocimiento que los futuros profesionales que ejerzan la profesión de periodistas han de tener respecto a otras comunicaciones afines con las que habrán de trabajar de forma cotidiana. El alumno debe "aprender a identificar correctamente mensajes de distinta naturaleza, analizarlos y conocer el proceso de realización de cada uno". (Universidad Rey Juan Carlos, 2020).

Por tanto, con la asignatura de "Procesos de Comunicación en Publicidad, Relaciones Públicas y Audiovisual”, "el alumno adquirirá un conocimiento del proceso de comunicación que se lleva a cabo en otras disciplinas afines con las que tendrá relación directa o, incluso, en las que podrá desempeñar su labor periodística". Pero también "aprenderá a analizar cualquier mensaje publicitario, corporativo y audiovisual a partir de los elementos que los integran. En este sentido, el contenido de la materia se concibe con un enfoque teórico y práctico, equilibrado y eminentemente profesional, de análisis y conocimiento actualizado de la actividad del profesional de la comunicación en distintos entornos laborales". (Universidad Rey Juan Carlos, 2020).

\section{CONTEXTO}

La presente práctica de innovación educativa docente, desarrollada durante el curso 2020-2021 en la asignatura "Sociología de la Desviación", vinculada a las materias básicas de la rama de Ciencias Sociales y Jurídicas, perteneciente al Grado de Criminología, Doble Grado en Criminología y Psicología, doble Grado en Criminología y Derecho y Doble Grado en Criminología y Trabajo Social de la Universidad Rey Juan Carlos. Se imparte en el primer cuatrimestre, con una carga lectiva de 6 CR ECTS, en segundo curso del Grado.

La muestra se compone de 127 alumnos evaluados. "Sociología de la Desviación" da continuidad a la asignatura "Introducción a la Sociología" que los alumnos cursan en su primer año del Grado, donde se imparten las temáticas básicas de la doctrina sociológica. Esta asignatura se basa en la premisa de ofrecer un aprendizaje más motivador y pedagógico, utilizando YouTube como herramienta de trabajo.

En connivencia con las premisas anteriores, también se ha aplicado esta práctica de innovación docente en la asignatura "Procesos de Comunicación en Publicidad, Relaciones Públicas y Audiovisual", correspondiente a los Dobles Grados en Periodismo-Derecho, Periodismo Economía, PeriodismoHistoria, y Periodismo-Ciencia Política y Gestión Pública de la Universidad Rey Juan Carlos. Esta materia se imparte en el primer cuatrimestre del primer curso de los mencionados Dobles Grados, con una carga lectiva de 6 CR ECTS.

El universo muestral se compone de 31 alumnos. La asignatura "Procesos de Comunicación en Publicidad, Relaciones Públicas y Audiovisual" se establece como una continuidad de sinergias conceptuales inherentes a otras materias como "Teorías de la Comunicación" y "Estructura del Sistema de Medios", correspondientes al itinerario formativo de los Dobles Grados mencionados.

Los objetivos principales de esta experiencia de innovación docente son los siguientes:

- Fomentar un acercamiento al uso de herramientas digitales dentro del aula.

- Estimular su motivación, generando interacción y colaboración entre los discentes de forma grupal, a 
través de la visualización de materiales en la plataforma audiovisual YouTube (películas y campañas publicitarias) directamente relacionadas con las asignaturas.

- Promover su actitud crítica y reflexiva.

- Conocer los fundamentos y procesos de producción específicos de la Publicidad, las Relaciones Públicas y la Comunicación Audiovisual.

\section{DESCRIPCIÓN}

La práctica de innovación educativa consta de las siguientes fases metodológicas:

- En la primera fase, el docente selecciona de la plataforma YouTube el material directamente relacionado con la asignatura. En la presente investigación es el concerniente a las campañas de publicidad de la Fundación de Ayuda contra la Drogadicción (FAD) y de la Dirección General de Tráfico (DGT), así como la proyección de los filmes "Ciudad de Dios" y "El Odio".

- En la segunda fase, el profesor expone el concepto de conducta desviada y sus diferentes acepciones, tipologías y perspectivas teóricas desde los clásicos hasta nuestros días. Igualmente, se explican las principales características de los anuncios de publicidad y las premisas pertinentes para la realización de una campaña de publicidad sugerente para los consumidores.

- En la tercera fase, los alumnos, por grupos establecidos por el docente, visualizan el material pertinente, analizando y relacionando el concepto básico de conducta desviada, con términos básicos como control social, agentes formales e informales, armonía, socialización, normas, derecho penal, delito o castigo, con las teorías sociológicas que recuerden y ver cómo se muestran los mencionados preceptos en las diferentes escenas de la película. Además, como paso previo al visionado de las campañas de publicidad, el profesor propone una lluvia de ideas a modo de "Brainstorming" acerca de los contenidos de dichas campañas. Este proceso genera en el alumnado motivación, reflexión, creatividad y la adquisición de técnicas de trabajo en grupo, así como la mejora en habilidades comunicativas tanto individuales como grupales.

- En la cuarta fase, una vez realizado el visionado tanto de las campañas de publicidad como de las películas seleccionadas, se analizan y comentan con el grupo. Se realiza un debate en el que se pregunta a los alumnos si conocen los filmes y las campañas publicitarias, a la vez que se les invita a que realicen las aportaciones que consideren oportunas (fundamentalmente, cómo se reflejan en las películas los diferentes conceptos sociológicos, qué campaña publicitaria les ha impactado más y qué valor aporta a la sociedad), estableciéndose así un debate constructivo con el profesor.

El material seleccionado por el profesor en la plataforma YouTube se expone a continuación:

- Película "Ciudad de Dios". Recuperado de: https://www.youtube.com/watch?v=HMYoqIAlYE

- Película "El Odio". Recuperado de: https://www.youtube.com/watch?v=85sEbY5BGz Q

- Campañas de publicidad de la Fundación de Ayuda contra la Drogadicción (FAD). Recuperado de: https://www.youtube.com/watch?v=Okob1EpCVN Y

- Campañas de publicidad de la Dirección General de Tráfico (DGT). Recuperado de:

https://www.youtube.com/watch?v=O58_7Tw9K $\mathrm{Uk}$

https://www.youtube.com/watch?v=ecx0St5cEeA

Así, Youtube se configura como una plataforma audiovisual pertinente, ya que los docentes tienen a su disposición una herramienta digital gratuita que facilita su enseñanza diaria. Además, se posibilita el rápido aprendizaje de los alumnos porque les permite reconocer, analizar y relacionar la teoría de las asignaturas tanto con los fenómenos sociales desviados dentro de un contexto determinado como con el análisis de las campañas publicitarias seleccionadas.

Igualmente, se motiva a los alumnos mediante un material audiovisual, ya que, actualmente, se encuentran inmersos en el denominado "aprendizaje audiovisual", circunscrito a la asimilación de conceptos mediante la imagen y el sonido. Esta metodología, ya instaurada en la mayoría de las Universidades, estimula el aprendizaje, inscribiéndolo en la denominada "cultura audiovisual", que constituye el "modus vivendi" de los discentes, alejándose de otras metodologías obsoletas utilizadas antaño.

Por tanto, YouTube se consolida como un recurso audiovisual pertinente de enseñanza-aprendizaje instaurado en las Universidades, con la finalidad de que tanto docentes como discentes puedan utilizarlo al estar disponible en abierto y sin restricciones de uso.

\section{RESULTADOS}

Visionadas las películas por los 127 alumnos, se les divide por grupos de investigación compuestos por 5 alumnos. A continuación, se les propuso realizar una selección de diferentes escenas de las películas en cuestión para su análisis y relación directa con los conceptos básicos de la materia y, en especial, el concepto de conducta desviada. Una vez relacionados los conocimientos de la asignatura con las diferentes escenas de las películas, se dispuso una puesta en común, dirigida por el docente, por parte de todos los grupos. En ella, los alumnos pudieron ejemplificar y aplicar la teoría de la asignatura a través de diferentes escenas de las películas. Mediante las siguientes calificaciones obtenidas por los alumnos que se exponen en la tabla 1, se muestra lo beneficiosa que ha sido esta experiencia de innovación docente para el alumnado. 
Tabla 1

Resultados de la asignatura Sociología de la Desviación

\begin{tabular}{lcr}
\hline Sociología de la Desviación & Notas Finales & \% \\
\hline No Presentado & 1 & $0.79 \%$ \\
Suspenso & 1 & $0.79 \%$ \\
Aprobado & 8 & $6.30 \%$ \\
Notable & 107 & $84.25 \%$ \\
Sobresaliente & 10 & $7.87 \%$ \\
Matrícula de Honor & 0 & $0 \%$
\end{tabular}

Como se observa en la tabla 1 , un $84,25 \%$ del alumnado ha sido calificado con Notable, lo que demuestra que la mayoría de los estudiantes han realizado con éxito la experiencia. También hay que destacar que, solo un $6,3 \%$ de los estudiantes, obtuvo Aprobado.

Una vez que los 31 alumnos realizaron el visionado de las campañas de publicidad, se procedió a la formación de grupos de discentes de 3 alumnos, con la finalidad de realizar un trabajo colaborativo en el que analizaron las campañas de publicidad tanto de la Fundación de Ayuda a la Drogadicción (FAD) como de la Dirección General de Tráfico (DGT). Sin duda, dos instituciones conocidas por el alumnado para la realización de las prácticas de la materia. En el exhaustivo análisis realizado, se estableció una aplicación de los conceptos aprendidos en la asignatura que sirvió como punto de partida para su aplicación a las campañas de publicidad mencionadas.

Posteriormente, guiados por el docente, se realizó una puesta en común por parte de los alumnos de cada una de las campañas de publicidad. Los resultados de esta experiencia de innovación docente han sido muy satisfactorios, tal como atestiguan las calificaciones obtenidas por el alumnado en la asignatura, reflejadas en la tabla 2 por nomenclatura y número de alumnos que han alcanzado la calificación correspondiente.

Tabla 2

Resultados de la asignatura Procesos de Comunicación en Publicidad, Relaciones Públicas y Audiovisual

\begin{tabular}{lcr}
\hline $\begin{array}{l}\text { Procesos de Comunicación en } \\
\text { Publicidad, Relaciones Públicas y } \\
\text { Audiovisual }\end{array}$ & Notas Finales & \% \\
\hline No Presentado & 1 & $3.23 \%$ \\
Suspenso & 1 & $3.23 \%$ \\
Aprobado & 0 & $0 \%$ \\
Notable & 8 & $25.80 \%$ \\
Sobresaliente & 19 & $61.29 \%$ \\
Matrícula de Honor & 2 & $6.45 \%$
\end{tabular}

La experiencia de innovación educativa ha resultado muy satisfactoria, refrendada por las premisas siguientes: el 6,45\% de los alumnos ha obtenido Matrícula de Honor, la máxima calificación posible; el $61,29 \%$ ha alcanzado Sobresaliente; y el $25,8 \%$ ha logrado Notable, tal como se muestra en la tabla 2.
Figura 1

Resultados del debate Sociología de la Desviación

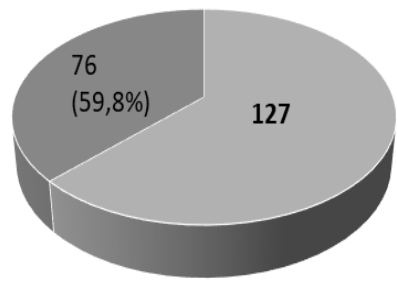

- Sociología de la Desviación

- № total de alumnos

- Alumnos que han aplicado adecuadamente las teorías sociológicas a las películas

Fuente: elaboración propia

Figura 2

Resultados del debate

Procesos de Comunicación en Publicidad, Relaciones Públicas y Audiovisual

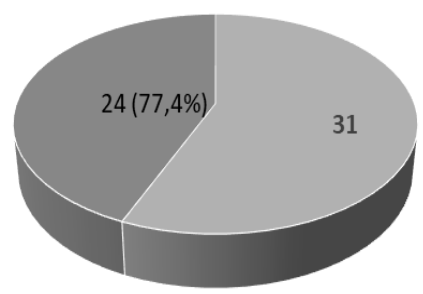

- Procesos de Comunicación en Publicidad, Relaciones Públicas y Audiovisuales

" № total de alumnos

- Alumnos con mayor impacto emocional por las campañas publicitarias de la DGT

Fuente: elaboración propia

A tenor del debate realizado con los alumnos tras la proyección tanto de las campañas publicitarias como de las películas mencionadas, 76 alumnos $(59,8 \%)$ de Sociología de la Desviación (Gráfico 1) analizaron correctamente las teorías sociológicas imbricadas en las escenas de los filmes, mientras que 24 alumnos $(77,4 \%)$ manifestaron su mayor impacto emocional por las campañas publicitarias de la Dirección General de Tráfico (DGT) (Gráfico 2). Por tanto, los datos precedentes indican que la experiencia de innovación docente realizada por parte del alumnado fue útil y beneficiosa, generando motivación y cooperación entre ellos, además de fomentar su capacidad crítica y de abstracción.

\section{CONCLUSIONES}

A raíz de la consolidación del Espacio Europeo de Educación Superior (EEES), la innovación docente se ha afianzado en el quehacer diario del profesorado universitario. Para ello, es fundamental la actitud, la aptitud y, sobre todo, la creatividad de los docentes a la hora de transmitir los conocimientos a los alumnos, así como empatizar con ellos. 
Del mismo modo, la presente experiencia de innovación docente es extrapolable a otras materias como "Introducción a la Sociología" y "Estructura Social Contemporánea", vinculadas al ámbito de la Sociología y, también, a "Teorías de la Comunicación" y "Estructura del Sistema de Medios", relacionadas con el ámbito de la Comunicación.

Por consiguiente, la presente innovación docente está orientada a mejorar el aprendizaje del alumnado como preparación para que adquiera las habilidades necesarias para enfrentarse, con suficientes garantías, a un mercado laboral complejo y en constante cambio. Resultados académicos muy satisfactorios, aprendizaje activo, trabajo colaborativo, creatividad, motivación, capacidad crítica, abstracción, empatía y resiliencia, entre otras premisas, capacidades y habilidades, se han consolidado como el núcleo vehicular concerniente a la puesta en marcha y desarrollo de esta práctica de innovación educativa cuya praxis metodológica continuará durante los próximos cursos, ampliándose a otras asignaturas asociadas a las áreas de conocimiento de Sociología y de Comunicación.

\section{REFERENCIAS}

Becker, H. (2010). Forasteros: hacia una sociología de la desviación. Buenos Aires, Argentina: Siglo XXI Iberoamericana.

Centro Nacional de Información y Comunicación Educativa (CNICE). Recuperado de:

http://recursos.cnice.mec.es/media/publicidad/bloque1/e mergentes/emep1_1b.html

Fanjul Fernández, M. y Morales Estévez, R. (2017). Introducción al comportamiento criminal: la conducta desviada. Recuperado de: https://es.eserp.com/wpcontent/uploads/2018/01/Gui\%CC\%81a-de-

Sociologi\%CC\%81a-de-la-Desviacio\%CC\%81n.pdf

Gallego Domínguez, C. y Murillo-Estepa, P. (2018). La práctica docente mediada con tecnologías: YouTube como herramienta de aprendizaje en educación superior. Foro educacional, (31), 11-29.

Maraza-Quispe, B., Alejandro-Oviedo, O., FernándezGambarini, W., Cisneros-Chavez, B., \& ChoquehuancaQuispe, W. (2020). Análisis de YouTube como herramienta de investigación documental en estudiantes de educación superior. Publicaciones, 50(2), 133-147. doi:10.30827/publicaciones.v50i2.13949

McQuail, D. y Windahl, S. (1997). Modelos para el estudio de la comunicación colectiva. Pamplona, España: EUNSA.

Muela, C. (2019). Procesos de Comunicación, en Publicidad, Relaciones Públicas y Audiovisual. Madrid, España: Síntesis.

Rivière, J. (2013). Interpretaciones del comportamiento desviado y control social. Recuperado de: http://openaccess.uoc.edu/webapps/o2/bitstream/10609/6 9967/4/Desviaci\%C3\%B3n\%20y\%20control\%20social portada.pdf

Universidad Rey Juan Carlos. (2020). Procesos de Comunicación en Publicidad, Relaciones Públicas y Audiovisual. Guía docente. Madrid: Universidad Rey Juan Carlos. Recuperado de:
file://C:/Users/Usuario/Downloads/GuiaDocente_PROC ESOS\%20DE\%20COMUNICACION\%20EN\%20PUBL ICIDAD, \%20RELACIONES\%20PUBLICAS\%20Y\%20 AUDIOVISUAL\%20(3).pdf

Vicente, M. (2011). Sociología de la Desviación: una aproximación a sus fundamentos. Alicante, España: Club Universitario. 